insects are not inconsistent with tropical insect communities exhibiting levels of population fluctuation comparable with temperate ones; the stabilising tendencies of such $\mathrm{K}$-ish life history strategies can easily be offset by the lower dynamical stability that is characteristic of more complex (species-rich) ecosystems. This view that tropical and temperate communities may manifest similar levels of $\mathrm{AV}$, accomplished by tropical populations exhibiting special features that help offset the intrinsically greater dynamical fragility of a complex system, is clearly a personal one, but it deserves to be considered.

In short, the body of information collected and compiled by Wolda suggets that tropical insect populations, including those from tropical forests, do not show systematically lower fluctuations in abundance than their temperate cousins. Insect populations from relatively dry areas, where the rainfall is low and unpredictable, do tend to fluctuate more; this tendency is, however, equally true for tropical and temperate insects.

\section{What's special about actin?}

from Michael Geisow

THERE is little doubt that actin enables cells literally to shape up to their role in an organised community. Even loners like the amoeba do not dispense with this ubiquitous protein. Although fibrous (F)-actin is most familiar as the protein component of the thin filaments of skeletal muscle, current interests centre on non-muscle actins which are probably present in the cytoplasm of all cells. In non-muscle cells, Factin forms the microfilaments frequently observed in association with the plasma membrane. Representative structures are the fibrous bundles within cell microvilli and stress fibres apparent in fibroblasts spread on a solid surface. These structures are frequently labile-the major distinction between muscle and non-muscle cells is that the latter have mechanisms for maintaining actin in its non-polymerised state (G-actin). These general observations raise three key questions. In non-muscle cells, what regulates the intracellular distribution of actin filaments and the degree of actin polymerisation? What determinants govern the attachment of actin to the plasma

Michael Geisow is a member of the scientific staff at the National Institute for Medical Research, London.

\title{
Third age of quantum chemistry
}

from Graham Richards

THE earliest quantum mechanical calculations of molecular properties were very crude and hoped only for agreement with experiment within an order of magnitude. The interest in this pioneering work arose from the fact that approximate agreement did provide some insight as to the reasons for the values of measured properties.

A second age depended on the advent of increasingly powerful computers. $A b$ initio calculations of molecular orbital wave functions boomed and continue to fill the scientific literature in many areas. These more accurate calculations are in much closer agreement with experimentally measured parameters. The complexity of the calculation often precludes the use of simple pictorial explanations and the answers cannot be preferred to experiment. Apart from proving the methods, this type of work receives most justification when applied to systems where the experiment is not a viable alternative to computation; perhaps the molecule is unstable or cannot be synthesised.

Now steady improvements in theoretical calculations have led to the dawning of a third age: calculations which are more accurate than experiment or at very least sufficiently accurate to be indispensible for interpretation. An example of such a case is the elegant spectroscopic experiment of Hunziker and his colleagues at the IBM Research Laboratory San Jose (Wendt, Hippler \& Hunziker J. chem. Phys. 70 in the press).

The experiment concerns the nearinfrared electronic absorption spectrum of the cis isomer of electronically excited triplet acetylene, formed by mercury-photosensitised reactions of acetylene, ketene and diazomethane. Although aliphatic unsaturated hydrocarbons in their lowest triplet electronic states are important chemical intermediates they have not been studied spectroscopically, in contrast to the widely investigated aromatic triplets. In its lowest triplet excited state acetylene adopts a planar bent

membrane and to itself to form microfilament bundles? And last, but most crucial, how are the assembled actin fibres used to generate the diverse types of motion (or tension) with which they are apparently associated?

Very often the most profitable lines of work in complex biological systems have tackled the molecular character of the protein responsible. It is therefore surprising that although many actins geometry with both hydrogens on the same side of the carbon-carbon bond (cis).

The key to identifying the spectrum was the theoretical work of Wetmore and Schaefer (J. chem. Phys. 69, 1678 ; 1978) which showed that the observed features correspond closely with the predictions for the ${ }^{3} \mathrm{~A}_{2}-$ ${ }^{3} \mathbf{B}_{2}$ transition of acetylene. The spectroscopists were able to observe rotational and vibronic structure as well as deuterium isotope effects, again agreeing to a remarkable degree with the $a b$ initio theoretical predictions.

An electric dipole transition between the triplet states is allowed for cis geometry $\left({ }^{3} \mathrm{~A}_{2}-{ }^{3} \mathrm{~B}_{2}\right)$ but parity forbidden in the trans case $\left({ }^{3} \mathrm{~A}_{u}-\right.$ $\left.{ }^{3} \mathbf{B}_{11}\right)$ and the two forms are separated by a potential barrier of the order of $1 \mathrm{eV}$. The spectrum was observed using a photochemical modulation apparatus. With this technique small transient absorptions are observed by phase sensitive detection and reaction rates determined using phase shift measurements. These showed that the lifetime of the transient at $1.35 \mu \mathrm{m}$ in the acetylene experiments was about $3 \times 10^{-5} \mathrm{~s}$. The principal feature of the spectrum is a single band at $1.35 \mu \mathrm{m}$ supporting both the calculations and spectroscopic analysis which show that the geometries of the two electronic states are very similar. There is for instance a change in $\mathrm{C}-\mathrm{C}-\mathrm{H}$ bond angle of about $2^{\circ}$.

The formation of the excited cis acetylene is particularly efficient using $\mathrm{Hg}$-photosensitised ketene and diazomethane. The suggested mechanism for this process involves the triplet state of methylene, $\mathrm{CH}_{2}$, in the reaction

The work represents perhaps a near perfect instance of theory being in harmony with experiment, each aspect vital to the other and the combination much more than the sum of the separate parts.

Graham Richards is a lecturer in the Physical Chemistry Laboratory, University of Oxford.

are readily purified in quantity, structural information is sparse. The few amino acid sequences which are known indicate that actin is very highly conserved; there are only 17 changes out of 375 amino acids between mammalian cytoplasmic actin and that isolated from the lower eukaryote Physarum polycephalum (Vanderkerchove \& Weber Nature 276, 720; 1978). Five of these differences occur at the 